\title{
BMJ Open A cross-sectional survey of awareness of human papillomavirus-associated oropharyngeal cancers among general practitioners in the UK
}

\author{
Matt Lechner, ${ }^{1,2}$ Claire Vassie, ${ }^{1,3}$ Cemal Kavasogullari, ${ }^{4}$ Oliver Jones, ${ }^{1}$ \\ James Howard, ${ }^{5}$ Liam Masterson, ${ }^{6}$ Tim Fenton, ${ }^{1}$ Wendell Yarbrough, ${ }^{7}$ Jo Waller, ${ }^{8}$ \\ Richard Gilson ${ }^{3}$
}

To cite: Lechner M, Vassie C, Kavasogullari C, et al. A cross-sectional survey of awareness of human papillomavirus-associated oropharyngeal cancers among general practitioners in the UK. BMJ Open 2018;8:e023339. doi:10.1136/ bmjopen-2018-023339

- Prepublication history and additional material for this paper are available online. To view these files, please visit the journal online (http://dx.doi. org/10.1136/bmjopen-2018023339).

ML, CV and CK contributed equally.

Received 5 April 2018 Revised 11 June 2018 Accepted 12 June 2018

Check for updates

(C) Author(s) (or their employer(s)) 2018. Re-use permitted under CC BY-NC. No commercial re-use. See rights and permissions. Published by BMJ.

For numbered affiliations see end of article.

Correspondence to

Dr Matt Lechner;

m.lechner@ucl.ac.uk and Dr

Richard Gilson;

r.gilson@ucl.ac.uk

\section{ABSTRACT}

Objectives To examine the level of awareness of the link between human papillomavirus (HPV) and oropharyngeal cancer (OPC) and epidemiological trends in HPV-related OPC among general practitioners (GPs) in the UK.

Design Cross-sectional survey.

Participants 384 GPs from England, Scotland, Wales and Northern Ireland.

Setting The survey was administered at GP training courses and via email to lists of training course attendees. Primary and secondary outcome measures Proportion of respondents aware of the link between HPV and OPC; respondents' self-rated knowledge of OPC; proportion of participants aware of the epidemiological trends in HPVassociated OPC.

Results 384 questionnaires were completed with an overall response rate of $72.9 \%$. $74.0 \%$ of participants recognised HPV as a risk factor for OPC, which was lower than knowledge about the role of smoking, chewing tobacco and alcohol consumption (all $>90 \%$ recognition). Overall, $19.4 \%$ rated their knowledge of OPC as very good or good, $62.7 \%$ as average and $17.7 \%$ as poor or very poor. The majority $(71.9 \%)$ were aware that rates of HPVassociated OPC have increased over the last two decades. Fewer than half $(41.5 \%)$ of the participants correctly identified being male as a risk factor of HPV-associated OPC, while $58.8 \%$ were aware that patients with HPVassociated OPC tend to be younger than those with nonHPV-associated disease.

Conclusions The association of HPV infection with OPC is a relatively recent discovery. Although the level of awareness of HPV and OPC among GPs was high, the recognised, indicating the need for further education.

\section{INTRODUCTION}

Head and neck cancer (HNC) is a significant cause of morbidity and mortality with over 11000 new cases diagnosed in the UK each year. ${ }^{1}$ The most common anatomical sites are the oral cavity, pharynx and larynx, with over $90 \%$ of tumours diagnosed being squamous cell carcinomas. ${ }^{2}$ Survival rates of HNC are characteristics of HPV-associated OPC were less well
Strengths and limitations of this study

- This study is the first to report on awareness of human papillomavirus (HPV)-associated oropharyngeal cancer (OPC) in a sample of UK general practitioners (GPS).

- This study included GPs from a variety of geographical regions with varying levels of experience.

- This study suggests that further education on the epidemiological trends and patient demographics of HPV-associated OPC may be needed to ensure early detection of disease.

- The main limitation is the unknown representativeness of the sample. Although the response rate was good, we used a convenience sample of GPs and trainees attending training updates, who may have higher or lower levels of knowledge than the wider population of GPs in the UK.

related to histological type, primary tumour site, disease stage at time of diagnosis and aetiology. Knowledge of the symptoms, risk factors and epidemiology both in the general population and among primary care professionals is key to primary prevention and earlier diagnosis. ${ }^{3}$

Research in the past decade has revealed the increasing importance of human papillomavirus (HPV) infection as a major risk factor for the development of $\mathrm{HNC}$, in particular oropharyngeal cancer (OPC). ${ }^{45}$ While rates of tobacco-related disease have decreased over the past two decades, there has been a marked increase in HPV-positive OPC in many high-income countries. It is estimated that between $55 \%$ and $95 \%$ of OPC in Europe is HPV-positive in younger patients. ${ }^{56}$ Twenty years ago, this figure stood at just $20 \%$. In this period, the incidence of HPV-associated OPC in the USA has increased by $225 \%{ }^{7}$ and similar trends are observed in the UK. 
In Scotland, the rates of OPC have increased more than those of any other cancer. ${ }^{8}$

Diagnosing HPV-positive OPC presents particular challenges for general practitioners (GPs) who may lack detailed knowledge of the disease. HPV-positive OPC represents a distinct molecular, epidemiological and clinical entity. ${ }^{910}$ Compared with non-HPV associated HNC, HPV-positive OPC occurs at a younger age and more often in men compared with non-HPV-associated OPC. ${ }^{11}$ In addition, patients presenting with HPV-positive OPC typically lack traditional risk factors such as smoking and alcohol use. Risk factors for HPV-positive OPC include age at first intercourse, number of sexual partners and number of oral sex partners. ${ }^{4}$ Early diagnosis is important; HPV-positive OPC is much more responsive to chemotherapy and radiotherapy ( $82 \%$ vs 55\%) and has better overall survival rates at 2 years than non-HPV-associated OPC $(95 \%$ vs $62 \%) .{ }^{12}$ The increasing rates of HNCs and the fact that their outcome depends on early diagnosis has been recognised in the GP Curriculum of the Royal College of General Practitioners. ${ }^{13}$

Awareness of the changing epidemiological and risk factor profile of OPC among primary care professionals is likely to lead to earlier diagnosis, resulting in improved outcomes, but the HPV-related learning resources recommended by the RCGP Curriculum to GPs and GP Trainees (such as RCGP Learning, e-LfH and BMJ Learning) focus on cervical cancer and cervical screening. Our review of the literature did not identify any learning modules available to UK GPs which focus on HPV-associated OPC. In addition, there has been no research to establish current levels of knowledge about HPV-associated OPC among GPs in the UK, which is an essential first step in identifying additional training needs in this area.

This study represents a first step towards understanding awareness of HPV-associated OPC among GPs in the UK, and to identify areas where further educational resources might lead to more targeted knowledge and improved patient outcomes.

\section{METHODS}

We carried out a cross-sectional questionnaire survey with GPs in the UK (online supplementary file 1; STROBE checklist).

\section{Measures}

We developed a short questionnaire (online supplementary file 2) assessing demographic characteristics of the participants, self-rated knowledge of OPC, awareness of OPC risk factors, awareness of time trends in smoking-related and HPV-related OPC, previous awareness of the link between HPV and OPC and perceptions about the characteristics of patients with HPV-associated as opposed to non-HPV OPC. Demographic characteristics included gender, number of years since graduation and current position. Self-rated level of knowledge and assessment of awareness of OPC was assessed using a Likert scale.
Knowledge of the symptoms and risk factors for OCP were assessed using items adapted from the Cancer Awareness Measure. ${ }^{1415}$ Thirteen risk factors (10 true and 3 false) were selected from the epidemiological literature. ${ }^{16}{ }^{17} \mathrm{An}$ open question was used to assess knowledge of the symptoms of OPC, using wording from the Cancer Awareness Measure. The items developed for the questionnaire were discussed in a multidisciplinary team including GPs and a behavioural scientist.

\section{Participants and procedure}

As the aims of the study were descriptive, we did not carry out a formal sample size calculation but aimed to recruit around 400 participants from a range of geographical locations, with varying amounts of clinical experience, in line with similar studies of this type ${ }^{18}$ Eligibility criteria were defined as being on the General Medical Council's (GMC) GP register and a local performers list or being currently enrolled in a recognised UK GP training post. The questionnaire was administered to a convenience sample of GPs attending courses and training meetings in paper format and via email lists in online format. Survey Monkey online platform was used to administer the survey via email lists. Paper questionnaires were added as separate collectors to the Survey Monkey database to consolidate responses. The sample included three training events for GPs in London and the North West of England, and five web links sent to working groups, local faculties and event administrators. Each collector was sent and tracked separately (online supplementary table 1; supplementary file 3). Data collection continued over a period of 9 months between May 2015 and February 2016.

\section{Analysis}

Statistical analysis was conducted using standard measures of central tendency and spread. One-way analysis of variance test was used for assessing association between years after graduation and awareness of HPV and OPC link. IBM SPSS V.20.0 software was used for statistical analysis. Descriptive data on the main characteristics of the sample have been analysed using Microsoft Excel V.2013.

\section{Patient and public involvement}

No patient and public involvement was sought during the preparation of the questionnaire, as our target group was exclusively medical professionals. However, GPs and a behavioural scientist were involved in the development of the survey who are included in the list of authors.

\section{RESULTS}

\section{Sample characteristics}

A total of 96 surveys were distributed in printed form and 411 requests were sent by email distribution via online lists. Overall, 385 questionnaires were completed giving an overall response rate of $72.9 \%$. Eight of the 385 responses were disqualified. A total of 340 participants completed all the questions and 35 has skipped one or 
Table 1 Demographic characteristics of the sample and self-rated knowledge of OPC $(n=376)$

\begin{tabular}{lcc}
\hline \multicolumn{1}{c}{$\mathbf{N}$} & $\%$ \\
\hline Gender & & \\
\hline Male & 154 & 40.9 \\
\hline Female & 222 & 59.1 \\
\hline Stage of training/position & \\
\hline F2 & 6 & 1.6 \\
\hline GPST1 & 44 & 11.7 \\
\hline GPST2 & 36 & 9.6 \\
\hline GPST3 & 49 & 13.1 \\
\hline GP (salaried or & 218 & 57.9 \\
partnered) & & \\
\hline Locum & 23 & 6.1 \\
\hline Years since graduation & & \\
\hline$<2$ & 17 & 4.4 \\
\hline 2-5 & 81 & 21.9 \\
\hline 5-10 & 93 & 24.2 \\
\hline 10-20 & 69 & 17.9 \\
\hline 20+ & 124 & 32.3 \\
\hline Location of current practice $(\mathrm{n}=373)$ & \\
\hline England & 165 & 44.2 \\
\hline Scotland & 103 & 27.6 \\
\hline Wales & 32 & 8.6 \\
\hline Northern Ireland & 73 & 19.6 \\
\hline Self-rated knowledge of OPC (n=350) & \\
\hline Very poor & 5 & 1.4 \\
\hline Poor & 57 & 16.3 \\
\hline Average & 220 & 62.9 \\
\hline Good & 65 & 18.6 \\
\hline Very good & 3 & 0.9 \\
\hline
\end{tabular}

GP, general practitioner; OPC, oropharyngeal cancer.

more questions. Each question was analysed individually. The response rate for paper questionnaires was higher $(85.4 \%)$ compared with online distribution (70.1\%) (online supplementary table 1; supplementary file 3). The demographic characteristics of the sample are shown in table 1 . Overall, $59.1 \%$ participants were women. The largest proportion of participants were from England $(44.2 \%)$, but all parts of the UK were included (Scotland $27.6 \%$, Ireland $19.6 \%$ and Wales $8.6 \%$ ). About half $(50.3 \%)$ of participants had been in practice for over 10 years $(18.4 \% 10-20$ years and $31.9 \%>20$ years $)$. Just over a third $(35.2 \%)$ of participants were in training, $33.5 \%$ were salaried GPs and $24.5 \%$ were GP partners, while $6.1 \%$ reported being in a locum post. When asked about their self-rated knowledge of OPC, $19.4 \%$ rated their knowledge of OPC as very good or good, $62.9 \%$ rated their knowledge as average and $17.7 \%$ reported poor or very poor knowledge of OPCs.

\section{Awareness of risk factors for OPC}

$73.9 \%$ of the participants reported being aware of the link between HPV and OPC while $16.9 \%$ reported not having heard of the association and $9.1 \%$ reported being unsure. Participants were asked about 13 exposures and whether they were risk factors of OPC or not (figure 1). Just over three-quarters $(77.6 \% 271$ of 349$)$ of the participants who responded to the risk factor question correctly identified HPV as a risk factor for OPC. Awareness of other well-established risk factors was much higher: smoking (99.4\%), chewing tobacco $(96.6 \%)$, current alcohol consumption $(94.3 \%)$ and past alcohol consumption (86.5\%). Chewing catchu and areca nuts, marijuana use and aflatoxin exposure were less frequently recognised $(32.9 \%, 50.4 \%$ and $26.4 \%$, respectively).

\section{Knowledge of the epidemiology of HPV-associated OCP}

Most participants $(71.9 \%)$ correctly stated that the rates of HPV-associated OPC have increased in high incomes countries over the last two decades (table 2). However, fewer than half $(41.5 \%)$ correctly identified HPV-associated OPC as being more common in men. A 58.8\% correctly reported the association with younger age. There were no statistically significant differences between years since graduation or post type and awareness of HPV and $\mathrm{OPC}$.

\section{DISCUSSION}

This study is the first to assess the awareness of HPV-associated OPC in a sample of UK GPs. Our results show that more than three-quarters of UK participants who responded to the risk factor question correctly identified HPV as a risk factor for OPC. However, there is limited awareness of the differences in the demographic profiles of patients presenting with HPV-associated and non-HPV-associated OPC as well as a lack of awareness of other risk factors of OPC.

Cancer of the oropharynx is now thought to be associated with HPV in 70\%-95\% of younger patients ${ }^{5}$ and disease outcome is related to the stage of disease at diagnosis. ${ }^{3}$ It is therefore important that GPs, who are likely to be the first point of contact for patients, are able to recognise the characteristics of patients at risk of HPV-associated OPC in order to refer them on to secondary and tertiary care as early as possible. These patients may be younger and may not have any history of smoking and drinking, in contrast to the risk factor profiles of patients with traditional HNC.

Our study demonstrates that further education on the epidemiological trends and patient demographics of HPV-associated OPC is needed to ensure early detection of disease. We provide a learning module which can be accessed online (online supplementary file 4; HPV-associated Oropharyngeal Cancer Module). GP awareness of the role of HPV in OPC may also lead to more support for the continued high uptake of national HPV vaccination 


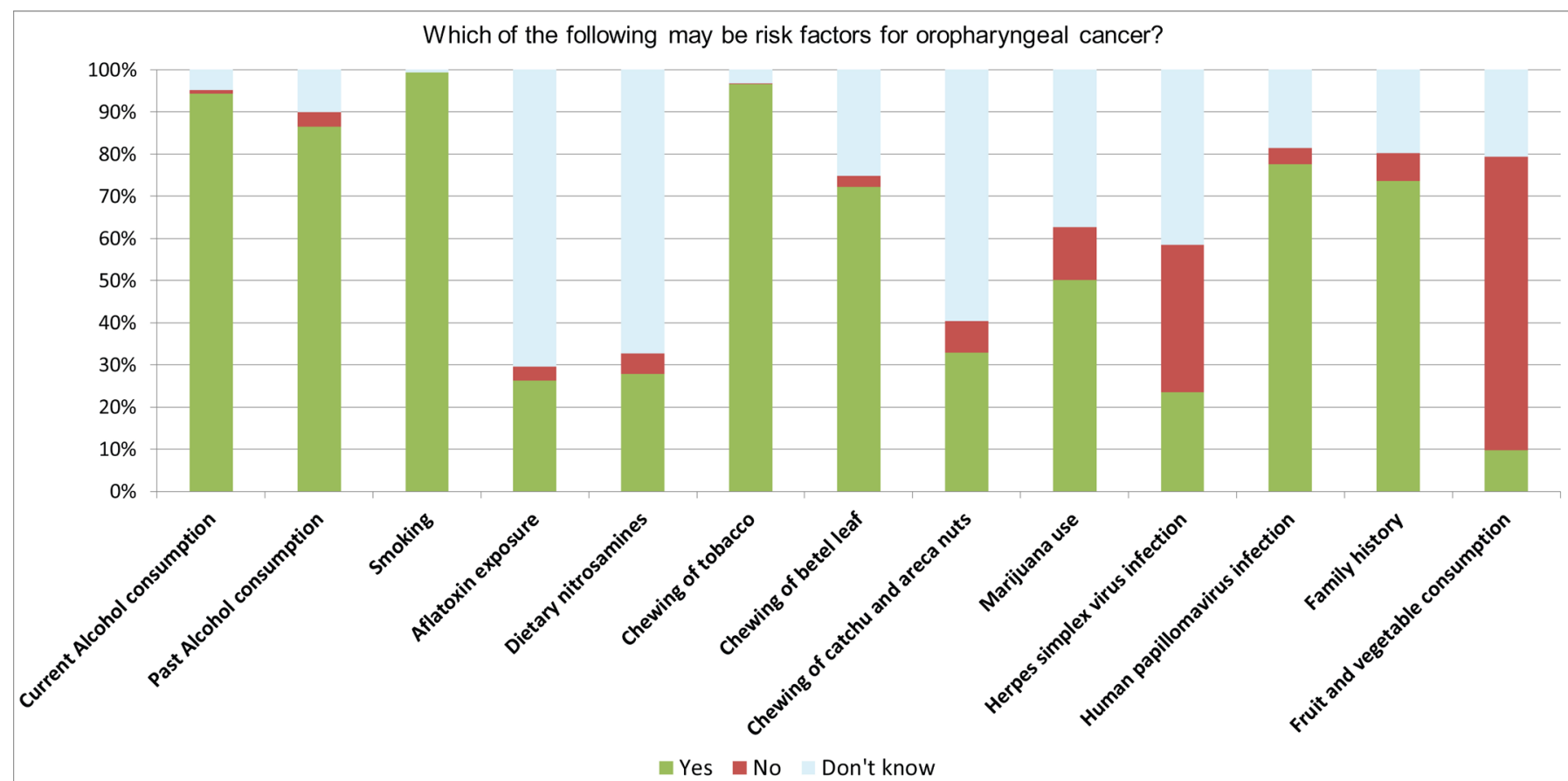

Figure 1 Participant responses to questions on 10 proven risk factors for OPC, and 3 factors which are not known risk factors. OPC, oropharyngeal cancer.

programmes, thereby maximising opportunity to ensure both individual and herd protection.

\section{Strengths and limitations}

Our study is the first to assess the awareness of HPV-associated OPC in a sample of UK GPs and benefited from the

Table 2 Knowledge about HPV-associated OPC $(n=342)$

\begin{tabular}{lcc}
\hline \multicolumn{4}{c}{$\mathbf{N}$} & $\%$ \\
\hline Aware of OCP-HPV link before today? & \\
\hline Yes & 253 & 73.9 \\
\hline No & 58 & 16.9 \\
\hline Not sure & 31 & 9.1 \\
\hline Over the last two decades, have rates of HPV-OPC \\
\hline Increased & 246 & 71.9 \\
\hline Decreased & 9 & 2.6 \\
\hline Stayed the same & 29 & 5.6 \\
\hline Do not know & 68 & 19.9 \\
\hline Compared with non-HPV-OPC, are HPV-OPC patients... \\
\hline Male & 142 & 41.5 \\
\hline Female & 74 & 21.6 \\
\hline Same gender & 43 & 5.2 \\
\hline Do not know & 83 & 21.9 \\
\hline Younger & 201 & 58.8 \\
\hline Older & 48 & 14.1 \\
\hline Same age & 18 & 5.3 \\
\hline Do not know & 75 & 21.9 \\
\hline
\end{tabular}

HPV, human papillomavirus; OPC, oropharyngeal cancer. inclusion of participants from all four UK nations. It is of immediate relevance, taking into account that it assesses awareness of a disease which shows a rapidly rising incidence and a changing epidemiology.

A total of 41985 GPs $(82.16 \%$ of the total number of registered GPs in the UK) were registered in England in September 2016, 4953 GPs, excluding locums, in Scotland in January 2017 (9.7\%), 2887 GPs in Wales, including 634 locums, in March 2016 (5.6\%) and 1274 GPs, excluding locums, in Northern Ireland in October $2015(2.49 \%)$, respectively. ${ }^{19}$ Comparing these data with our sample, we need to acknowledge that participants from England $(\mathrm{n}=165 ; 44.2 \%)$ are under-represented and that participants from Scotland $(\mathrm{n}=103 ; 27.6 \%)$, Wales $(\mathrm{n}=32 ; 8.6 \%)$ and Northern Ireland $(\mathrm{n}=73 ; 19.6 \%)$ are over-represented. We instructed all participants to answer the questions consecutively when distributing the paper questionnaires, but they may have looked at subsequent questions which could have influenced their responses leading to an overestimation of knowledge. The online questionnaire only allowed questions to be answered in order. Although the response rate was high for a survey of GPs, ${ }^{20}$ we do not have data on non-responders so we are unable to test for response bias. The main limitation of the study is the use of a convenience sample, recruited at GP educational events, and via email lists of event attendees and regional faculties. By recruiting participants attending training courses and educational events, we may have included GPs who were more up-todate, leading to an overestimation of knowledge levels. Training events included GP related topics in general and were not related to head and neck pathology or HPV. In 
addition, we developed some of the questionnaire items specifically for the study and although they were reviewed by the study team and had good face validity, further psychometric validation would be appropriate prior to further use.

\section{Comparison with the existing literature}

A recent systematic review by Dodd et al evaluated the psychosocial impact of HPV-related HNC and investigated the awareness of the link between HPV and HNC among different populations. Forty-one studies were identified which measured knowledge of the link between HPV and $\mathrm{HNC}$, demonstrating the lowest level of knowledge in the general population and highest in medical and dental professionals. ${ }^{21}$ However, Signorelli et al showed that only $38 \%$ of Italian GPs $(n=938)$ recognised the role of HPV in oral disease and oral cancer and concluded that there is a lack of knowledge on HPV infection and vaccination in Italian GPs. ${ }^{22}$ Odone et al explored reasons for non-vaccination against HPV in Italy, ${ }^{23}$ providing a useful basis to plan, implement and evaluate targeted educational programmes and training. The link between HPV and OPC was recognised by $43.3 \%$ of primary care physicians in Jordan $^{24}$ and by $54 \%$ of a sample of German physicians. ${ }^{25}$

A study of 2126 adults in the USA demonstrated that knowledge of HNC among the general public is low. While $54 \%$ of participants identified smoking as a risk factor for HNC, $4.8 \%$ identified alcohol use and just $0.8 \%$ recognised HPV infection as a risk factor. ${ }^{14}$ Family physicians could play a key role in educating the public and encouraging HPV vaccine uptake.

We report findings of the first study investigating the awareness of HPV-associated OPC risk factors in a sample of UK GPs. Awareness in the UK is high but there are gaps in knowledge that should be addressed.

\section{Implications for practice}

The failure of $41.2 \%$ of participants to recognise younger age as a characteristic of HPV-related OPC patients should be addressed. Good awareness of the fact that there has been a significant rise in younger patients presenting with OPC, often lacking a history of smoking and high alcohol intake, will help ensure that HPV-associated OPC is recognised early. Younger patients with HPV-associated OPC often present with asymmetrical tonsils and without knowledge of this disease and its presentation in the absence of classical risk factors makes it likely that early diagnosis is missed.

Our results clearly demonstrate the need for awareness campaigns to make UK GPs more aware of this rapidly evolving disease. We have created a learning module which can be freely accessed online (online supplementary file 4; HPV-associated Oropharyngeal Cancer Module). This includes reference to other risk factors that we showed to be frequently underrecognised, such as chewing betel nuts (also referred to as catchu or areca nuts) which are traditional among some communities in Indian, Pakistan and Bangladesh.
In conclusion, this study suggests a clear need for further education for GPs on a disease which shows a rising incidence and a changing epidemiology .

Author affiliations

${ }^{1}$ UCL Cancer Institute, University College London, London, UK

${ }^{2} \mathrm{Head}$ and Neck Centre, University College London Hospitals NHS Trust, London, UK ${ }^{3}$ Institute for Global Health, University College London, London, London, UK ${ }^{4} \mathrm{GP}$, West of Scotland, Glasgow, UK

${ }^{5}$ ENT Department, Wrexham Maelor Hospital, Wrexham, UK

${ }^{6}$ Department of Otolaryngology, Lister Hospital, Stevenage, UK

${ }^{7}$ Department of Surgery, Division of Otolaryngology, Yale School of Medicine, New Haven, Connecticut, USA

${ }^{8}$ Research Department of Behavioural Science and Health, University College London, London, UK

Acknowledgements The authors would like to thank the general practitioners who took part in the study for their time and effort in completing the surveys and to the educational bodies who gave us permission to distribute questionnaires at GP training events.

Contributors ML, CV, CK, OJ, RG: wrote the first draft of the manuscript with contributions from JH, TF, LM, JW and WY. CV, CK, JH: collected the data and analysed these under the guidance of WY, RG and ML. OJ and ML: created the online module with advice from LM and TF.

Funding This research received no specific grant from any funding agency in the public, commercial or not-for-profit sectors.

Competing interests None declared.

Ethics approval The study was exempted from the requirement for Research Ethics Committee (REC) review on the basis that data collection was anonymised and no vulnerable participants were involved (advice from Harrow NHS REC and UCL REC).

Provenance and peer review Not commissioned; externally peer reviewed.

Data sharing statement All data from this study are included in the present work. Open access This is an open access article distributed in accordance with the Creative Commons Attribution Non Commercial (CC BY-NC 4.0) license, which permits others to distribute, remix, adapt, build upon this work non-commercially, and license their derivative works on different terms, provided the original work is properly cited, appropriate credit is given, any changes made indicated, and the use is non-commercial. See: http://creativecommons.org/licenses/by-nc/4.0/.

\section{REFERENCES}

1. Cancer Research UK. Head and neck cancer survival statistics. 2017 http://www.cancerresearchuk.org/health-professional/cancerstatistics/statistics-by-cancer-type/head-and-neck-cancers/survival.

2. Hunter KD, Parkinson EK, Harrison PR. Profiling early head and neck cancer. Nat Rev Cancer 2005;5-127-35.

3. Pai SI, Westra WH. Molecular pathology of head and neck cancer: implications for diagnosis, prognosis, and treatment. Annu Rev Pathol 2009;4:49-70.

4. D'Souza G, Kreimer AR, Viscidi R, et al. Case-control study of human papillomavirus and oropharyngeal cancer. $N$ Engl J Med 2007;356:1944-56.

5. D'Souza G, Dempsey A. The role of HPV in head and neck cancer and review of the HPV vaccine. Prev Med 2011;53 Suppl 1(Suppl 1):S5-S11.

6. Schache AG, Powell NG, Cuschieri KS, et al. HPV-Related Oropharynx Cancer in the United Kingdom: An Evolution in the Understanding of Disease Etiology. Cancer Res 2016;76:6598-606.

7. Chaturvedi AK, Engels EA, Pfeiffer RM, et al. Human papillomavirus and rising oropharyngeal cancer incidence in the United States. $J$ Clin Oncol 2011;29-4294-301.

8. Association BD. Oral and Oropharyngeal Cancer fact sheet for healthcare professionals, 2017.

9. Gillison ML. Human papillomavirus-associated head and neck cancer is a distinct epidemiologic, clinical, and molecular entity. Semin Oncol 2004;31-744-54.

10. Lechner M, Fenton TR. The Genomics, Epigenomics, and Transcriptomics of HPV-Associated Oropharyngeal Cancer-- 
Understanding the Basis of a Rapidly Evolving Disease. Adv Genet 2016;93:1-56.

11. Chaturvedi AK. Epidemiology and clinical aspects of HPV in head and neck cancers. Head Neck Pathol 2012;6 Suppl 1(Suppl 1):16-24.

12. Fakhry C, Westra WH, Li S, et al. Improved survival of patients with human papillomavirus-positive head and neck squamous cell carcinoma in a prospective clinical trial. $J$ Nat/ Cancer Inst 2008;100:261-9.

13. The RCGP Curriculum: Professional \& Clinical Modules (Version approved 19 January 2016 for implementation from 1 February 2016). https://www.gmc-uk.org/RCGP_Curriculum_modules jan2016.pdf_68839814.pdf2016.

14. Luryi AL, Yarbrough WG, Niccolai LM, et al. Public awareness of head and neck cancers: a cross-sectional survey. JAMA Otolaryngol Head Neck Surg 2014;140:639-46.

15. Stubbings S, Robb K, Waller J, et al. Development of a measurement tool to assess public awareness of cancer. Br J Cancer 2009;101 Suppl 2(Suppl 2):S13-S17.

16. Gupta B, Johnson NW. Emerging and established global life-style risk factors for cancer of the upper aero-digestive tract. Asian Pac J Cancer Prev 2014;15:5983-91.

17. Pytynia KB, Dahlstrom KR, Sturgis EM. Epidemiology of HPVassociated oropharyngeal cancer. Oral Oncol 2014;50:380-6.
18. Dodd RH, Forster AS, Waller J, et al. Discussing HPV with oropharyngeal cancer patients: A cross-sectional survey of attitudes in health professionals. Oral Oncol 2017;68:67-73.

19. BMA. General practice in the UK - background briefing, 2017.

20. Huddlestone L, Walker GM, Hussain-Mills R, et al. Treating tobacco dependence in older adults: a survey of primary care clinicians' knowledge, attitudes, and practice. BMC Fam Pract 2015;16:97.

21. Dodd RH, Waller J, Marlow LA. Human Papillomavirus and Head and Neck Cancer: Psychosocial Impact in Patients and Knowledge of the Link - A Systematic Review. Clin Oncol 2016;28:421-39.

22. Signorelli C, Odone A, Pezzetti F, et al. [Human Papillomavirus infection and vaccination: knowledge and attitudes of Italian general practitioners]. Epidemiol Prev 2014;38(6 Suppl 2):88-92.

23. Odone A, Visciarelli S, Lalic T, et al. Human papillomavirusassociated cancers: a survey on otorhinolaryngologists knowledge and attitudes on prevention. Acta Otorhinolaryngol Ital 2015;35:379-85.

24. Hassona Y, Scully C, Shahin A, et al. Factors Influencing Early Detection of Oral Cancer by Primary Health-Care Professionals. $J$ Cancer Educ 2016;31:285-91.

25. Hertrampf K, Wenz HJ, Koller M, et al. Knowledge of diagnostic and risk factors in oral cancer: results from a large-scale survey among non-dental healthcare providers in Northern Germany. $J$ Craniomaxillofac Surg 2014;42:1160-5. 